\title{
A Simple Spectropolarimetric Temperature Diagnostic for Circumstellar Disks
}

Karen S. Bjorkman \& Jon E. Bjorkman

Ritter Observatory, Dept. of Physics $\&$ Astronomy, University of Toledo, Toledo, $\mathrm{OH}$ 43606, USA

Kenneth Wood

Smithsonian Astrophysical Observatory, 60 Garden St., Cambridge, MA 02138 USA

\begin{abstract}
We describe a technique for estimating average temperatures of Be star disks from analysis of ultraviolet spectropolarimetry. The technique utilizes the fact that the spectrum of the scattered starlight is sensitive to the circumstellar opacity, and hence temperature, since the signature of the disk material is imprinted on the scattered light spectrum. Analysis of the polarization spectrum thus allows us to disentangle the relative contributions of the star and disk, and thereby obtain an estimate of the average disk opacity as a function of wavelength. Using an LTE line-blanketed model (containing about $10^{6}$ spectral lines) for the opacity, we determine a theoretical opacity as a function of temperature. By comparing this to the opacity deduced from the spectropolarimetry, we can estimate the average disk temperature. For classical Be stars, the relative strengths of the $\mathrm{Fe}$ II and Fe III multiplets at around $2400 \AA$ and $1900 \AA$, respectively, are a sensitive temperature diagnostic, so that the temperature estimate can be made within $\pm 1000 \mathrm{~K}$. We demonstrate our technique with analysis of UV spectropolarimetry (from WUPPE) of the classical Be star $\zeta$ Tau, for which we infer an isothermal disk temperature of $14000 \mathrm{~K}$.
\end{abstract}

\section{Introduction}

Spectropolarimetric observations of Be stars in both optical and ultraviolet (UV) wavelength ranges are now more widely available. Recent high-quality optical data have been obtained with the Halfwave Polarimeter (HPOL) at the Pine Bluff Observatory (PBO), and UV data were obtained with the Wisconsin Ultraviolet Photo-Polarimeter Experiment (WUPPE) during the Astro-1 and Astro-2 missions. Figure 1 shows an example (for $\zeta$ Tau) of the complete range of data now available for a number of Be stars.

Earlier models including only hydrogen opacity were able to fit existing optical spectropolarimetric data rather well; such analysis can provide information about the disk geometry and density (Wood, Bjorkman, \& Bjorkman 1997). However, it has been clear for some time that simple hydrogen-only models do not adequately explain the UV spectropolarimetric observations obtained by 


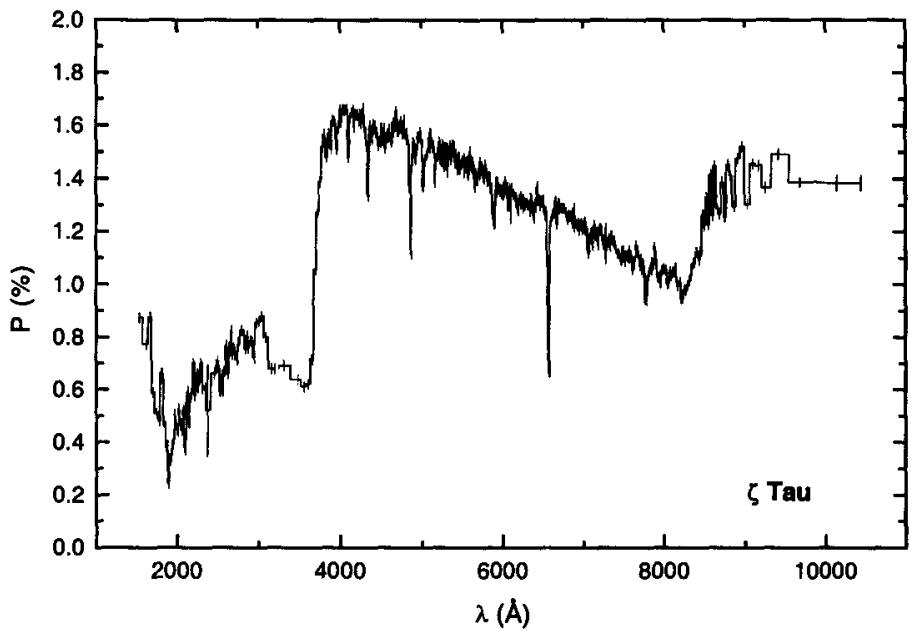

Figure 1. The polarization data for $\zeta$ Tau from the UV to the nearIR. (Adapted from Wood et al. 1997)

WUPPE (Bjorkman et al. 1991; 1993). The UV observations show pronounced line-blanketing depolarization due to the presence of substantial opacity from metal lines (Bjorkman et al. 1991), and the line blanketing shifts from Fe III to Fe II as the temperature decreases. Theoretical models of LTE line opacity show that the shift from Fe III to Fe II occurs at a temperature of about $13000 \mathrm{~K}$. This effect provides a means of using UV spectropolarimetry to diagnose temperatures within the disk itself.

\section{Method}

We have used theoretical models of LTE line opacity to compare with an "effective opacity" derived from the spectropolarimetric observations. The polarization level depends on the opacity of the disk as a function of wavelength, since the polarized light is attenuated by its passage through the disk.

To obtain the effective opacity as a function of wavelength, we employed a simple model in which we assumed that (at each wavelength) the albedo was a constant independent of position in the disk. We then used a Monte Carlo model (Wood et al. 1996) to determine the polarization, $P$, as a function of albedo, $a=\sigma_{e} /\left(\kappa+\sigma_{e}\right)$, for several inclination angles. For each inclination angle, we normalized $P(a)$ by its maximum, $P_{\max }=P(1)$. By doing this, we effectively remove the inclination angle dependence, and the normalized polarization is approximately the same for all inclination angles. A simple fit to the Monte Carlo results gives

$$
P / P_{\max }=1.22 a-0.22 a^{2} .
$$




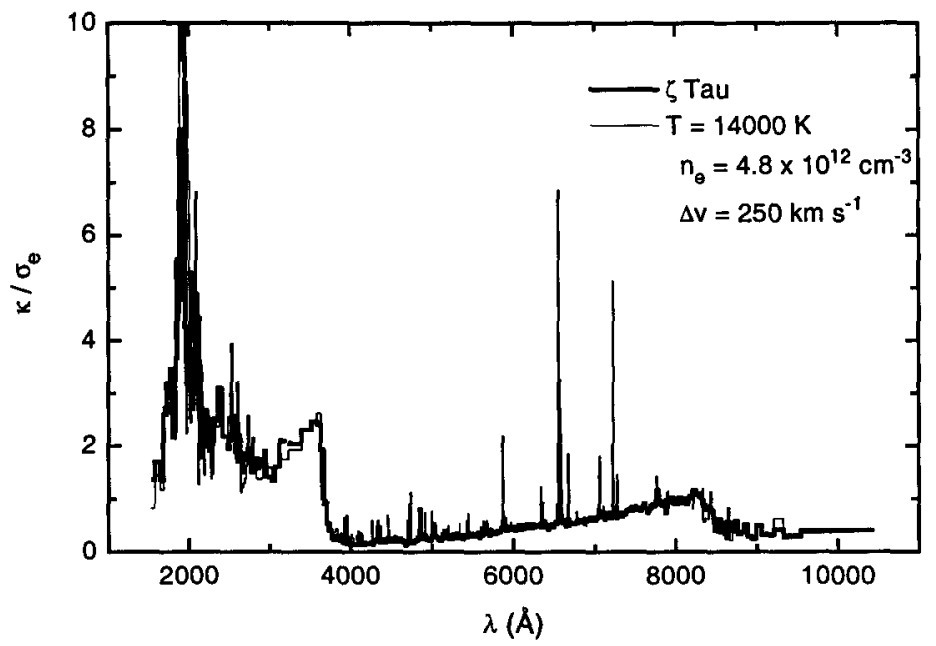

Figure 2. The best fit model for the effective opacity (determined from the polarization). The bold line is the opacity derived from the spectropolarimetry, while the thin line is the best fit model, at an average disk temperature of $14000 \mathrm{~K}$.

Inverting this equation, we find that the albedo as a function of wavelength is given by

$$
a(\lambda)=\frac{2 P(\lambda) / P_{\max }}{1.22+\sqrt{1.448-0.88 P(\lambda) / \bar{P}_{\max }}}
$$

Figure 2 shows the opacity, $\kappa / \sigma_{e}=(1 / a)-1$, obtained from the polarization shown in Figure 1. Overplotted on this is the best fit theoretical model, derived as follows.

For the theoretical opacity, LTE level populations were calculated using partition functions from Traving, Baschek, \& Holweger (1966), utilizing computational routines from the TLUSTY program (Hubeny, private communication). Spectral line data (about $10^{6}$ lines) were taken from the Kurucz (1998) line lists. The lines were broadened using a "square" line profile function, $\phi_{\nu}$, with an effective velocity width $\Delta v$ to account for rotation and expansion in the disk:

$$
\phi_{\nu}=\frac{1}{\Delta \nu}=\frac{\lambda}{\Delta v}
$$

The albedo is averaged over each wavelength bin (denoted by index $i$ )

$$
\left\langle a_{i}\right\rangle=\frac{1}{\Delta \lambda} \int_{\lambda_{i}}^{\lambda_{i}+\Delta \lambda} \frac{1}{1+\kappa / \sigma_{e}} d \lambda
$$

where $\kappa$ is the total line opacity, summed over all lines. Note that the average albedo is equivalent to using the Rosseland mean opacity. 
This theoretical opacity is then compared to that obtained from the polarization observations. The temperature was varied to get the best fit to the observations. The primary temperature indicators were the Fe III line blends at around $1900 \AA$ and the Fe II line blends from $2400-2600 \AA$. By matching these two regions, we were able to determine a temperature to within about $1000 \mathrm{~K}$. Since we have assumed a constant albedo with position in the disk, the derived temperature represents a sort of global average temperature of the material in the disk. Since the polarization is produced within only a few stellar radii, this temperature is probably dominated by material relatively close to the star.

\section{Conclusions}

In the case of $\zeta$ Tau, we estimate an average disk temperature of $14000 \mathrm{~K}$. By comparison, the stellar temperature is around $19000 \mathrm{~K}$ (Wood et al. 1997), so we find that $T_{\text {disk }} \approx 0.75 T_{\text {eff }}$.

Acknowledgments. We thank Marilyn Meade, Brian Babler, and Ken Nordsieck for assistance with data calibration and reduction. This work has been supported in part under NASA grants NAG5-3447 and NAG5-8054 (KSB) and NAG5-3248 (JEB) to the University of Toledo. KW acknowledges support from NASA grant NAG5-6039. Spectropolarimetric observations with WUPPE and HPOL were supported under NASA contract NAS5-26777 with the University of Wisconsin. K.S. Bjorkman is a Cottrell Scholar of the Research Corporation, and gratefully acknowledges their support.

\section{References}

Bjorkman, K.S. et al. 1991, ApJ 383, L67

Bjorkman, K.S. et al. 1993, ApJ 412, 810

Kurucz, R. 1998, http://craku5.harvard.edu/linelists/gfall/

Traving, G., Baschek, B., Holweger, H. 1966, in Abhandlungen aus der Hamburger Sternwarte, Bd. VII, Nr. 1, T. 1 (Hamburg: Hamburger Sternwarte), p. 25.

Wood, K., Bjorkman, K.S., Bjorkman, J.E. 1997, ApJ 477, 926

Wood, K., Bjorkman, J.E., Whitney, B.A., Code, A.D. 1996, ApJ 461, 847 